\title{
BMJ Open Assessment of patient experience profiles and satisfaction with expectations of treatment effects by using latent class analysis based on a national patient experience survey in Taiwan
}

Shang-Jyh Chiou, ${ }^{1}$ Pei-Chen Lee, ${ }^{1}$ Yu-Hsuan Chang, ${ }^{2}$ Pei-Shan Huang, ${ }^{2}$ Li-Hui Lee, ${ }^{1}$ Kuan-Chia Lin ${ }^{3}$

To cite: Chiou S-J, Lee P-C, Chang $\mathrm{Y}-\mathrm{H}$, et al. Assessment of patient experience profiles and satisfaction with expectations of treatment effects by using latent class analysis based on a national patient experience survey in Taiwan. BMJ Open 2019;9:e023045. doi:10.1136/ bmjopen-2018-023045

\section{- Prepublication history and} additional material for this paper are available online. To view these files, please visit the journal online (http://dx.doi. org/10.1136/bmjopen-2018023045).

Received 21 March 2018 Revised 13 December 2018 Accepted 24 January 2019

Check for updates

(C) Author(s) (or their employer(s)) 2019. Re-use permitted under CC BY-NC. No commercial re-use. See rights and permissions. Published by BMJ.

${ }^{1}$ Health Care Management, National Taipei University of Nursing and Health Sciences, Taipei, Taiwan

2Planning, National Health Insurance Admission, Taipei, Taiwan

${ }^{3} \mathrm{Hospital}$ and Health Care Administration, National YangMing University, Taipei, Taiwan

Correspondence to

Dr Shang-Jyh Chiou; shangjyh@ntunhs.edu.tw

\section{ABSTRACT}

Objectives Health system responsiveness is a complicated issue that guides researchers wishing to design an efficient methodology for enhancing understanding of perspectives regarding healthcare systems. This study examined the relationship between patient experience profiles and satisfaction with expectations of treatment effects.

Design This was a cross-sectional study. We used eight items obtained from latent class analysis to develop patient experience profiles.

Setting Primary care users in Taiwan.

Participants This study conducted an annual National Health Insurance survey in Taiwan and sampled from those who had experience with the medical service in primary care clinics in 2015.

Primary outcome measure Respondents were asked to indicate the extent of their satisfaction with their expectation of treatment effects (or symptom improvement).

Results The proportions of participants in groups $1-4$ were $34 \%, 24 \%, 29 \%$ and $12 \%$, respectively. Patients in good health were more satisfied with their expectations of treatment effects (OR 1.639, $p=0.007$ ). Furthermore, group 4 (-eAll) were less satisfied with their expectations of treatment effects than those in the other three groups (ORs: group 1 (+eAll): 9.81 , group 2 (-CwR): 4.14 and group 3 $(-\mathrm{CnR}): 4.20)$.

Conclusions The results revealed that experiences of poor accessibility and physician-patient relationships affected the patients' expectations. Therefore, greater accessibility and more positive physician-patient relationships could lead to higher patient satisfaction with their expectations of treatment effects. Furthermore, the findings could assist authorities in targeting specific patients, with the objective of improving their healthcare service experience. They could also serve as a mechanism for improving the quality of healthcare services and increase accountability in healthcare practices.
Strengths and limitations of this study

- This is a national survey data regarding the patient experience in primary care setting.

- However, we cannot guarantee the findings represent the entire population.

- Latent class analysis (LCA) is a novel approach in patient experience for the advantage of conceptualising the study framework.

- Currently, using the cross-validation technique to confirm the validity from LCA model is hard in this study.

\section{INTRODUCTION}

WHO in 2000 stated that health systems have three fundamental objectives: improving health, meeting patients' expectations and providing financial protection. ${ }^{1}$ Health system responsiveness is related to the manner and environment in which individuals are treated. Analysis of health system responsiveness can result in improvements in care quality within the system and increased healthcare options based on individuals' experiences. ${ }^{2}$ Responsiveness in different healthcare domains ${ }^{34}$ is a complex issue that requires identification of efficient methodology to facilitate health officials' understanding of the public's perspective of the health system. Therefore, a structured, participant-orientated approach is required to consider several domains and related survey items simultaneously.

Several factors, including healthcare outcomes and quality, influence patients' expectations of healthcare services. To enhance understanding of the factors that determine healthcare outcomes, researchers typically use the Andersen behavioural 
model $^{5}$ to construct and explain factors in the predisposing, enabling and need dimensions. Patients' experiences and outcomes are important in their own right. ${ }^{6-8}$ In most situations, patients' experiences and satisfaction are based on outcomes ${ }^{9}$ and the fulfilment of their expectations and vice versa. Moreover, numerous factors influence patients' healthcare experiences ${ }^{10}{ }^{10}$ which affect their satisfaction levels. For instance, some studies have indicated that different health statuses are associated with different satisfaction levels, ${ }^{11}$ and outcomes for patients with sufficient medical resources were typically more positive relative to those of patients with insufficient resources. ${ }^{12-14}$ In addition, patient characteristics, such as demographic factors and health beliefs, ${ }^{15}$ could be positively or negatively associated with healthcare utilisation, which is related to satisfaction levels. Therefore, an understanding of these characteristics could aid the development of useful strategies to improve system performance and quality.

Greater patient satisfaction is usually positively associated with higher healthcare quality ${ }^{16}$ and positive patientphysician interactions ${ }^{17}$ when patients receive healthcare services. Health authorities desire to collect information from the patient report experience system to improve the healthcare system by responding to people's expectations. Based on an annual national survey, health officials often exhibit great interest in using self-report measures to assess patients' experiences, particularly those involving patient satisfaction. This information helps them to improve the quality of healthcare service delivery and evaluate the performance of healthcare systems. However, researchers usually face two dilemmas when analysing self-reported data from national survey databases. The first dilemma involves the representativeness of the sample. ${ }^{18} 19$ Fortunately, there are complicated statistical tools available, which can be used to find solutions, even when a sample is imperfect and beyond the scope of a study. ${ }^{20}{ }^{21}$ The second dilemma involves the challenge of understanding factors that influence patient satisfaction with healthcare providers and systems, which is due to the complexity of the data, such as factors pertaining to patients' characteristics, health behaviours and status that can be collected from patient surveys. Therefore, researchers face difficult decisions such as those regarding the number of variables that should be included in a model to predict satisfaction or system performance. Hence, the use of different methods is required to address this issue.

One such method is latent class analysis (LCA), which can be used to create patient experience profiles within a participant-orientated approach, depending on the purpose of the study. LCA has been used widely in the social sciences ${ }^{22}$ during the last two decades. It is a mixed model used for examining unobserved categorical variables and divides populations into different exclusive latent classes. ${ }^{23}$ For example, socioeconomic status (SES) includes educational level, income, occupation and family size, and each of these dimensions reflects only partial concepts within SES rather than the entire factor. Therefore, researchers use indicators as manifest SES variables and develop LCA models to identify unobservable subgroups within populations. In addition, LCA is used in the measurement of behaviour to enhance understanding of risk profiles for specific behavioural outcomes. ${ }^{24}$ In brief, using indicators directly in a given model reflects a variable-oriented method (eg, regression analysis). LCA is a participant-oriented method for classifying participants into different subgroups according to factor loadings or item response probability.

To date, numerous studies have discussed the factors related to patient satisfaction or patient experience using traditional variable-oriented methods; however, only a limited number of researchers have adopted a participant-oriented method (such as LCA) for evaluating patient satisfaction or experience. Further, most of these studies either had a small sample size or obtained data from only a limited number of institutions instead of using national survey data. Many countries have created agencies to administer national surveys regularly, because of increasing interest in collecting information regarding patients' experiences, to improve healthcare quality and system performance. ${ }^{25}{ }^{26}$ For example, in Taiwan, the National Health Insurance (NHI) administration has conducted national surveys annually for a decade.

The purpose of this study was to examine the relationship between respondents' patient experience profiles and their satisfaction with expectations of treatment effects. We collected data from the NHI annual survey, which was adopted from the Andersen behavioural model in question construction, in which items are associated with different patient experience profiles that could influence patients' satisfaction with expectations of treatment effects. In addition, we used a participant-oriented method, LCA, to understand discrepancies in patient experience profiles in primary care settings and retain as much measurability as possible in the observation setting. Our results can help health authorities improve the performance of health systems from the perspective of a patient's experience in primary care.

\section{METHODS}

\section{Population and setting}

This study used the annual NHI survey to explore patients' experiences of receiving medical services at a primary care clinic in 2015. The survey used the probability-proportional-to-size sampling approach, and random digit dialling was used in computer-assisted telephone interviewing (CATI). The survey was conducted from 24 August to 6 September 2015, and the target population included individuals who had received primary care services during the preceding 3 months. Parent proxy-reports were obtained for participants younger than 15 years of age. The final effective sample size was 2009, and the sampling error was $\pm 2.2 \%$. The response rate was around $9.6 \%$ in the CATI system. 


\section{Measurement instrument and LCA model}

The annual survey used a questionnaire consisting of six sections: (1) medical care utilisation and accessibility, (2) referral and medication compliance, (3) satisfaction and treatment effects evaluation, (4) health behaviour, (5) social network and (6) demographic characteristics. In accordance with Andersen's behavioural model, we initially included 10 items pertaining to patients' experiences, namely, time (travel times, waiting times and time spent in consultation), costs, self-evaluation or patient-evaluation (on receiving health education, easy-to-understand explanations and scheduling appointments), and health behaviour (drug compliance, alcohol use and tobacco use), in the LCA model. Basically, we assumed that a better patient experience (steps are taken to ease the geographical and financial barriers and physician-patient communication) and health behaviour (no drinking, no smoking and better drug compliance) led to increased overall satisfaction in the expectations of treatment outcome. The items regarding time included 'The last time you received primary care (within the last 3 months), how much time did you spend in one-way travel, including waiting for vehicles, traffic, and so on?' and 'Do you feel that the travel time was too long?' The items regarding cost included 'How much did you pay for primary care at your last consultation, including out-of-pocket fees?' and 'Do you consider this inexpensive or expensive?' The items regarding patient evaluation included 'When you need to obtain primary care, is it easy to make an appointment in advance?' The items regarding health behaviour included 'Did you complete the course of medication according to the provider's instructions?'

We could only include dichotomous items in the LCA model. Initially, we assessed the model's fitness and identified two items (scheduling appointments and drug compliance) with little discriminatory ability. When either or both items were excluded, the final classified pattern did not alter, and only the entropy value decreased from 0.79 to 0.71 . To ensure that the LCA model was concise, we included only eight items. The Akaike information criterion, Bayesian information criterion and entropy value for the LCA were 419.21, 615.39 and 0.71 , respectively, indicating that the LCA model was acceptable for use in further analysis. The details of the model selection procedure are given in the online supplementary file 1.

\section{Dependent variables}

The dependent variable in the study was satisfaction with expectations of treatment effects. Respondents were asked to indicate the extent of their satisfaction with expectations of treatment effects (or symptom improvement) using a Likert scale ranging from 1 (very satisfied) to 5 (very dissatisfied). Thereafter, we used their responses to categorise their satisfaction levels as high or low (neither satisfied nor dissatisfied, somewhat dissatisfied and very dissatisfied).

\section{Independent variables}

To improve understanding of factors associated with participants' satisfaction with expectations of treatment effects, we used the latent categorical factor identified in the LCA (ie, the four groups) and included sex, age, educational level, monthly income, marital status, living conditions, chronic diseases, catastrophic illness and health status. Note that the NHI in Taiwan issues beneficiaries a catastrophic illness certificate to exempt them from copayments if they qualify. To obtain information regarding health status, we asked participants to rate their health status during the preceding month. In addition, to obtain information regarding chronic diseases, we asked participants whether they had been diagnosed with hypertension, diabetes, hyperlipidaemia, asthma or heart disease. However, we did not count the numbers of chronic diseases and only used chronic disease (yes/no), and catastrophic illness (yes/no) in the later analysis.

Regarding missing values, every item was given a 'refuse to answer' in the CATI system when the participant did not answer this question. For monthly income, approximately $30 \%$ did not respond. For other items, less than $1 \%$ were not answered.

\section{Patient involvement}

No patients were involved in the analysis or interpretation of the results in this study nor the writing of any drafts. There are no plans to disseminate the results of this study to participants or any relevant patient networks.

\section{Statistical analysis}

We obtained the results of the LCA and deconstructed the four groups. We displayed the participants' characteristics (such as demography, living conditions, chronic diseases and health status) by group to depict the basic features of each group. Thereafter, a logistic regression model was developed to model patients' satisfaction with expectations of treatment effects (ie, high or low) as a function of the four groups identified via the LCA and various demographic factors. We also run another logistic regression for the potential misclassification inherent in two-step methods that weighted posterior probabilities of class membership based on LCA as a sensitivity analysis in online supplementary file 2 . From the result, the posterior probability of class membership using in the logistic regression produces the outcomes are similar to the original model. SAS V.9.3.1 (SAS Institute) and SPSS V.20.0 (SPSS) were used to perform all statistical analyses. The significance level was set at 0.05 .

\section{RESULTS}

The LCA model identified four groups (table 1): individuals who reported a positive experience of the receipt of healthcare services (group 1: +eAll); individuals who reported a negative experience of the receipt of healthcare services in communication and exhibited risky behaviour (group 2: -CwR); individuals who reported a 
Table 1 Results from LCA

\begin{tabular}{|c|c|c|c|c|c|}
\hline $\begin{array}{c}\text { AIC } \\
\text { BIC } \\
\text { Entropy }\end{array}$ & & $\begin{array}{l}419.21 \\
615.39 \\
0.71\end{array}$ & & & \\
\hline Proportion of population in each group & Total & $\begin{array}{l}1 \\
0.3436\end{array}$ & $\begin{array}{l}2 \\
0.2442\end{array}$ & $\begin{array}{l}3 \\
0.2886\end{array}$ & $\begin{array}{l}4 \\
0.1236\end{array}$ \\
\hline The travel time is not too long & $1878(93.8)$ & 0.9521 & 0.9733 & 0.9606 & 0.7753 \\
\hline The waiting time is not too long & $1574(78.3)$ & 0.8154 & 0.8586 & 0.8979 & 0.3045 \\
\hline The consulting time felt more satisfied & $1619(80.6)$ & 0.9771 & 0.9314 & 0.9750 & 0.7393 \\
\hline The feeling in expenditure is not high & $772(38.4)$ & 0.9242 & 0.8943 & 0.9314 & 0.6680 \\
\hline Received patient education in most time & $775(38.6)$ & 0.9441 & 0.0703 & 0.0795 & 0.0675 \\
\hline Received easy-to-understand explanation in most time & $1238(61.7)$ & 0.9991 & 0.4894 & 0.4209 & 0.2935 \\
\hline No drinking behaviour & $959(47.7)$ & 0.4789 & 0.0072 & 0.8471 & 0.5402 \\
\hline No smoking behaviour & $1558(77.6)$ & 0.7838 & 0.5337 & 0.9508 & 0.8305 \\
\hline
\end{tabular}

Group 1: positive experience in all (+eAll).

Group 2: negative experience in communication with risk health behaviour (-CwR).

Group 3: negative experience in communication without risk health behaviour (-CnR).

Group 4: negative experience in all (-eAll).

Bold values in 1, 2, 3, 4 are refering to each group from LCA analysis.

AIC, Akaike information criterion; BIC, Bayesian information criterion; LCA, latent class analysis.

negative experience of the receipt of healthcare services in communication without risk behaviour (group 3: -CnR) and individuals who exhibited self-dissatisfaction and reported an unsatisfactory experience of the receipt of healthcare services (group 4: -eAll). The proportions of participants in groups $1-4$ were $34 \%, 24 \%, 29 \%$ and $12 \%$, respectively. In group 1 (+eAll), almost six items reflected a positive response in their experience (eg, did not travel too long to their primary care, more satisfied with their primary care consulting time and received adequate patient education in primary care). Fewer respondents reported that the duration of the consultation with a primary care provider was too long or medical costs were too high; in addition, they reported they usually received patient education and easy-to-understand explanations in the primary care setting. In groups 2 and 3, medical costs and the durations of the consultations with primary care providers were rated positively; however, both groups reported negative experiences but usually received patient education and easy-to-understand explanations. Group 2 (-CwR) reported higher levels of drinking or smoking relative to the other three groups. Group 4 (-eAll) reported a higher number of negative experiences in the primary care setting relative to those reported by the other three groups.

Table 2 shows participants' characteristics according to group. Unsurprisingly, sex, age, educational level, monthly family income, marital status, chronic diseases and health status differed among the four groups, with living conditions and catastrophic disease being insignificant factors. Group 3 (-CnR) included higher proportions of women and older individuals and lower proportions of individuals who had been educated to a level above that of college, were married and had a higher health status relative to those in the other three groups; group 4's mean age was lower and the participants reported higher educational and income levels and lower health status relative to those of the other three groups.

The logistic model revealed significant differences in patients' health status and satisfaction with expectations of treatment effects across the four groups as shown in table 3. Patients with a more favourable health status held higher satisfaction with expectations of treatment effects (OR 1.639, $\mathrm{p}=0.007$ ). Furthermore, group 4 (-eAll) held lower satisfaction expectations of treatment effects relative to those reported by the other three groups (ORs: group 1 (+eAll): 9.81, group 2 (-CwR): 4.14, group 3 (-CnR): 4.20).

\section{DISCUSSION}

Significant differences in expected treatment effects were observed across patient experience profiles based on healthcare experiences and risky behaviour. Regardless of whether they exhibited health-related risky behaviour, participants who reported negative experiences with healthcare services expected the worst outcomes. The results showed that accessibility affected satisfaction with expectations of treatment effects, even those with NHI, which tends to reduce financial and geographical barriers. Most importantly, the receipt of health education or easy-to-understand explanations in the clinical setting also affected satisfaction with expectations of treatment effects. Healthcare system administrators should consider ways in which communication with health providers, improved health literacy and patient-doctor interpersonal relationships could help patients to understand situations involving their health. 
Table 2 Participants' characteristics in different groups from LCA

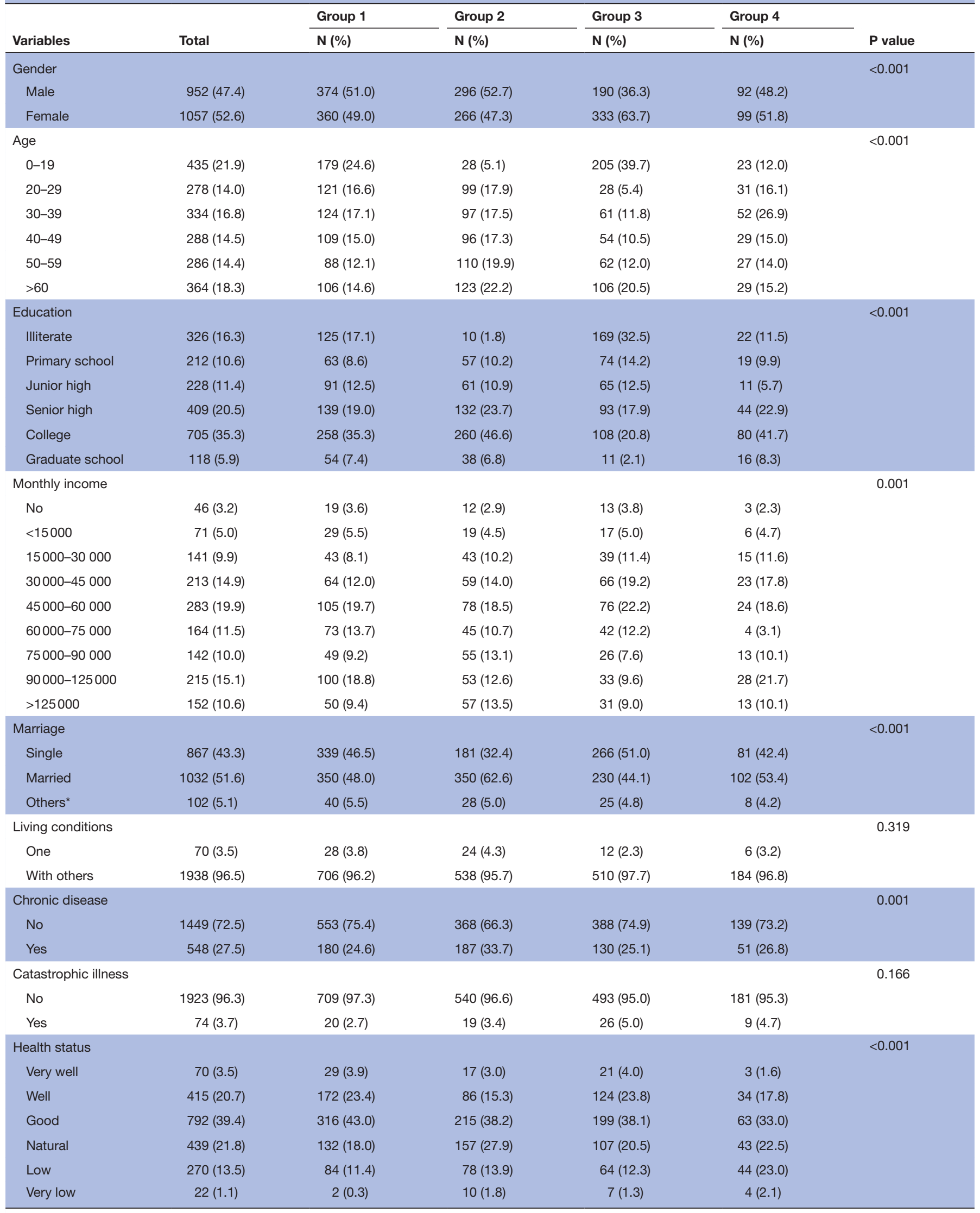

*Divorced and widowed.

LCA, latent class analysis. 
Table 3 The logistics model of satisfied expectations regarding treatment effects in different groups from LCA with participant characteristics

\begin{tabular}{lcccc}
\hline & & $95 \% \mathrm{Cl}$ & & \\
Variables & OR & Low level & Up level & P value \\
\hline $\begin{array}{lllll}\text { Gender } \\
\quad \text { Female }\end{array}$ & & & & 0.792 \\
Age & 1.05 & 0.73 & 1.50 & \\
$0-19$ & & & & 0.310 \\
$20-29$ & & & & \\
$30-39$ & 1.10 & 0.47 & 2.57 & \\
$40-49$ & 2.00 & 0.78 & 5.13 & \\
$50-59$ & 1.52 & 0.55 & 4.18 & \\
$>60$ & 2.29 & 0.83 & 6.34 & \\
& 2.25 & 0.83 & 6.11 &
\end{tabular}

\begin{tabular}{|c|c|c|c|c|}
\hline Education & & & & 0.883 \\
\hline Illiterate & & & & \\
\hline Primary school & 0.89 & 0.38 & 2.09 & \\
\hline Junior high & 0.87 & 0.39 & 1.96 & \\
\hline Senior high & 1.10 & 0.49 & 2.48 & \\
\hline College & 0.88 & 0.38 & 2.01 & \\
\hline Graduate school & 0.69 & 0.25 & 1.87 & \\
\hline Monthly income & & & & 0.327 \\
\hline$<45000$ & & & & \\
\hline $45000-59999$ & 1.50 & 0.87 & 2.59 & \\
\hline $60000-89999$ & 1.15 & 0.68 & 1.93 & \\
\hline$\geq 90000$ & 0.92 & 0.57 & 1.49 & \\
\hline Marriage & & & & 0.298 \\
\hline Single & & & & \\
\hline Married & 0.63 & 0.34 & 1.19 & \\
\hline Others & 0.89 & 0.31 & 2.58 & \\
\hline Living conditions & & & & 0.125 \\
\hline Living conditions with others & 1.98 & 0.83 & 4.75 & \\
\hline Chronic disease & & & & 0.217 \\
\hline Having chronic diseases & 1.34 & 0.84 & 2.13 & \\
\hline Catastrophic illness & & & & 0.504 \\
\hline Having catastrophic illness & 0.74 & 0.31 & 1.79 & \\
\hline Health status & & & & 0.007 \\
\hline Health status better & 1.64 & 1.15 & 2.34 & \\
\hline Group & & & & $<0.001$ \\
\hline Group 4 (-eAll) & & & & \\
\hline Group 1 (+eAll) & 9.81 & 5.70 & 16.89 & \\
\hline Group 2 (-CwR) & 4.14 & 2.53 & 6.77 & \\
\hline Group $3(-\mathrm{CnR})$ & 4.20 & 2.43 & 7.27 & \\
\hline
\end{tabular}

Group 1: positive experience in all (+eAll)

Group 2: negative experience in communication with risk health behaviour $(-\mathrm{CwR})$.

Group 3: negative experience in communication without risk health behaviour $(-\mathrm{CnR})$.

Group 4: negative experience in all (-eAll).

LCA, latent class analysis.

For analytical purposes, we included eight items in the LCA model based on the three components of the structure that reflects patients' experiences of the healthcare delivery system and their health-related risky behaviour.
The first component was accessibility. For instance, patients reported the durations (ie, long or short) of their travel times to consultations, waiting times for consultations and consultations with doctors. In addition, one item pertaining to medical expenditure (ie, high or low) measured their experience of financial barriers to accessibility. The responses to the items above reflect important decision points associated with satisfaction with expectations of final treatment effects. Previous research has shown that financial burden had a negative effect on patients' perspectives of their healthcare ${ }^{27}$ and that timelines were the most influential determinant of these perspectives ${ }^{28}$ and accessibility. ${ }^{1629}$

The second component was information received from care providers (or interpersonal communication), which is another important measure of patients' experiences. We assessed patients' reports regarding the receipt of patient health education or easy-to-understand explanations during consultations (ie, whether this usually occurred or did not usually occur) to determine whether the receipt of more information in the clinical setting was associated with higher satisfaction with expectations of treatment effects. Several studies have reported that positive interpersonal relationships and communication were associated with high-quality service and patient satisfaction. ${ }^{30-32}$

The third component was risky behaviour, such as drinking and smoking. Although very few studies have examined the relationship between health-related risky behaviour and patient satisfaction with expectations of treatment effects, we assumed that participants who exhibited health-related risky behaviour would have lower satisfaction with expectations of treatment effects. Another important consideration is that information regarding engagement in health-related risky behaviour is important in constructing patient experience profiles. However, some caveats should be considered in drawing this conclusion; in particular, the survey did not capture a complete profile of health-related risky behaviour or of the population engaging in it. Further investigation is required to confirm this assumption.

The Organisation for Economic Co-operation and Development developed a Healthcare Quality Indicators questionnaire and the Hospital Consumer Assessment of Healthcare Providers and Systems survey conducted in the USA indicated that patient experience is an important tool that serves as a surrogate marker for the quality and value of healthcare delivery. ${ }^{33}$ In Taiwan, based on the NHI annual survey, we categorised participants into four patient experience profile groups based on LCA and explored differences in satisfaction with expectations of health outcomes among the groups after adjusting for demographic and socioeconomic factors. Participants who reported a more favourable health status and positive patient experiences and did not exhibit health-related risky behaviour (ie, alcohol or tobacco use) tended to be more satisfied with their treatment effects. These findings are consistent with those of previous studies. Unsurprisingly, satisfaction with treatment effects could 
be enhanced by improving patients' experiences of healthcare services and patient-physician relationships in primary care settings.

Measuring patients' experiences has become a useful tool in the assessment of the quality of healthcare, particularly since the Affordable Care Act was passed in 2010. ${ }^{35} 36$ Positive patient experiences are important indicators of healthcare processes and outcomes,${ }^{32}$ which include good clinical outcomes, patient adherence to medical advice and low levels of healthcare utilisation. Some studies have shown a mixed association between patient experience and clinical processes and outcomes, which is unsurprising. ${ }^{38}$ In addition, numerous factors other than patient experience influence processes and outcomes. This is one reason why combining patient experience measures with other measures of healthcare quality is critical in creating an overall picture of performance.

Traditionally, when multiple variables are used to explore patients' experiences and satisfaction, they include age, sex, health status and health behaviour, among others. Researchers typically consider regression towards the mean and perform complicated modelling when more than 20 or 30 variables are examined. In addition, studies use multilevel models to address variation resulting from differences between doctors and patients, ${ }^{39}$ apply difference-in-difference analysis to perform evaluations within different time frames, ${ }^{40}$ or implement multimethod programmes to measure and improve patients' experiences. ${ }^{6}$ However, few studies have included patients who could be described using several behavioural variables. Moreover, a previous study showed that measurement often restricts evaluations to only one or two domains within a broader analysis of process or outcome measures. ${ }^{41}$ These restrictions result from the so-called variable-oriented approach. Therefore, we used LCA to examine the relationship between patients' experiences and treatment effects.

The study was subject to some limitations. For example, we were sceptical of the representativeness of the national survey, even after using appropriate weighting methods. Individuals in certain occupations and women are most likely to receive survey calls; therefore, we referred to the distribution of outpatient services in the NHI claim system to apply weights to the entire annual survey database. Although this weighting system was suitable, we cannot guarantee that the findings represent the entire population. In addition, LCA is a novel approach to the development of patient experience profiles, and it could serve as a powerful tool to explain subgroup behaviour. However, we should be mindful of the fact that it is sometimes difficult to obtain favourable results in patient experience profiles generated via LCA, particularly when some item responses are not easily distinguishable among different subgroups. For example, in this study, the ease of appointment scheduling and drug compliance lacked discrimination in the subgroups; thus, we decided to eliminate those items from the LCA model. Third, the patient experience measurements in NHI annual survey only reflect their general experience due to considering loading in limited time. Participants may have varied experience feeling in multiple physician office visiting; however, it is hard to catch the details in the NHI annual survey for determining the relationship with factors. Fourth, in some cases, participants' health statuses were in a period of transition, such as attending cancer therapy during the study period, which may have affected their final response regarding their satisfaction in expectations of treatment effects. This is a situation we have to consider the causal pathway between exposure and outcome. However, patients in Taiwan seeking primary care are usually not in acute care or in a stable chronic disease situation. In addition, the healthcare system does not have a gatekeeper design of primary care, and patients are not prevented from going directly to upper level hospitals. When a patient's health status undergoes a great change, that patient usually visits an upper level hospital for treatment. Those who underwent a great transition in health status may have represented only a small population and did not strongly influence the results of the study. Finally, patients with worse treatment outcomes may lead to report the negative healthcare experience. ${ }^{42}$ In this study, the cross-section data are hard to distinguish this kind of relationship. We have examined the reasons for not satisfied and only 38 of them $(n=110)$ were related to the negative treatment effect and need further study to provide more evidences.

\section{CONCLUSION}

Based on a participant-oriented perspective and analytical design, LCA was beneficial because it facilitated conceptualisation of the study framework and the simplification of the survey items. Our findings showed that greater accessibility and positive interpersonal relationships with healthcare providers could improve patients' satisfaction with expectations of treatment effects. These results could aid authorities in targeting specific patient groups to improve their experiences of healthcare services. Moreover, negative experiences of accessibility and physician-patient relationships influenced patients' satisfaction with expectations of treatment effects. This finding could serve as a mechanism to improve the quality of healthcare services and increase accountability in healthcare practices.

Acknowledgements This study was supported by NHI grant (2015): 'The study on Iongitudinal monitoring system of the right to seek medical care under the National Health Insurance' awarder by the National Health Administration.

Contributors S-JC and K-CL conceived and designed the study with additional inputs from P-CL and L-HL. K-CL, P-SH and Y-HC supervised the overall conduct and data collection process for the study. P-CL and S-JC managed the data set and analysed it with additional inputs from L-HL and K-CL. S-JC wrote the initial draft of the manuscript. All authors contributed to the critique and modification of the manuscript, read and approved the final version.

Funding National Health Insurance Administration with grant number MOHW104-NHI-S-114-112010.

Disclaimer The funder had no role in study design and analysis or decision to publish. 
Competing interests None declared.

Patient consent for publication Not required.

Ethics approval The Institutional Review Board (IRB) of the National Taiwan University has approved this study (201505ES011).

Provenance and peer review Not commissioned; externally peer reviewed.

Data sharing statement № additional data are available.

Open access This is an open access article distributed in accordance with the Creative Commons Attribution Non Commercial (CC BY-NC 4.0) license, which permits others to distribute, remix, adapt, build upon this work non-commercially, and license their derivative works on different terms, provided the original work is properly cited, appropriate credit is given, any changes made indicated, and the use is non-commercial. See: http://creativecommons.org/licenses/by-nc/4.0/.

\section{REFERENCES}

1. WHO. The world health report 2000. Health systems: improving performance. Geneva: World Health Organization, 2000.

2. Valentine NB, Bonsel GJ, Murray CJ. Measuring quality of health care from the user's perspective in 41 countries: psychometric properties of WHO's questions on health systems responsiveness. Qual Life Res 2007;16:1107-25.

3. Robone S, Rice N, Smith PC. Health systems' responsiveness and its characteristics: a cross-country comparative analysis. Health Serv Res 2011;46:2079-100.

4. Hsu CC, Chen L, Hu YW et al. The dimensions of responsiveness of a health system: a Taiwanese perspective. BMC Public Health 2006;6:72.

5. Andersen RM. Revisiting the behavioral model and access to medical care: does it matter? J Health Soc Behav 1995;36:1-10.

6. Burt J, Campbell J, Abel G, et al. Improving patient experience in primary care: a multimethod programme of research on the measurement and improvement of patient experience: Southampton (UK), 2017.

7. Ahmed F, Burt J, Roland M. Measuring patient experience: concepts and methods. Patient 2014;7:235-41.

8. Europe PI. Picker institute Europe's response to the department of health's consultation on the NHS outcomes framework. England: Picker Institute Europe, 2014.

9. Manary MP, Boulding W, Staelin R, et al. The patient experience and health outcomes. N Engl J Med 2013;368:201-3.

10. Bleich SN, Ozaltin E, Murray CK. How does satisfaction with the health-care system relate to patient experience? Bull World Health Organ 2009;87:271-8.

11. Otani K, Shen Y, Chumbler NR, et al. The impact of self-rated health status on patient satisfaction integration process. J Healthc Manag 2015;60:205-18.

12. Hawthorne TL, Kwan MP. Using GIS and perceived distance to understand the unequal geographies of healthcare in lower-income urban neighbourhoods. Geogr J 2012;178:18-30.

13. Raivio R, Jääskeläinen J, Holmberg-Marttila $D$, et al. Decreasing trends in patient satisfaction, accessibility and continuity of care in finnish primary health care - a 14-year follow-up questionnaire study. BMC Fam Pract 2014;15:98

14. Cabrera-Barona P, Blaschke T, Kienberger S. Explaining accessibility and satisfaction related to healthcare: a mixed-methods approach. Soc Indic Res 2017;133:719-39.

15. Wendt C, Kohl J, Mischke M, et al. How do Europeans perceive their healthcare system? patterns of satisfaction and preference for state involvement in the field of healthcare. Eur Sociol Rev 2010;26:177-92.

16. Al-Abri R, Al-Balushi A. Patient satisfaction survey as a tool towards quality improvement. Oman Med J 2014;29:3-7.

17. Gómez G, Aillach E. Ways to improve the patient-physician relationship. Curr Opin Psychiatry 2013;26:453-7.

18. Stone AA, Shiffman S. Capturing momentary, self-report data: a proposal for reporting guidelines. Ann Behav Med 2002;24:236-43.
19. Tsai AC, Chang TL. Quality issues of self-report of hypertension: analysis of a population representative sample of older adults in Taiwan. Arch Gerontol Geriatr 2012;55:338-42.

20. SH T, WY L, Hung YT. A study of survey nonresponse bias using propensity score adjustment. Political Science Review 2009;41:151-76.

21. Lin HW, Chen $\mathrm{YH}$. Adjustment for missing confounders in studies based on observational databases: 2-stage calibration combining propensity scores from primary and validation data. Am J Epidemiol 2014;180:308-17.

22. Savage $M$, Devine $F$, Cunningham $N$, et al. A new model of social class? findings from the bbc's great british class survey experiment. Sociology 2013;47:219-50.

23. GOODMAN LEOA. Exploratory latent structure analysis using both identifiable and unidentifiable models. Biometrika 1974;61:215-31.

24. Lanza ST, Rhoades BL. Latent class analysis: an alternative perspective on subgroup analysis in prevention and treatment. Prev Sci 2013;14:157-68.

25. Coulter A. Measuring what matters to patients. BMJ 2017;356:j816.

26. OECD. Recommendations to OECD ministers of health from the high level reflection group on the future of health statistics: Strengthening the international comparison of health system performance through patient-reported indicators: Organisation for Economic Co-operation and Development, 2017.

27. Chino F, Peppercorn J, Taylor DH, et al. Self-reported financial burden and satisfaction with care among patients with cancer. Oncologist 2014;19:414-20.

28. Mathews $M$, Ryan $D$, Bulman $D$. What does satisfaction with wait times mean to cancer patients? BMC Cancer 2015;15:1017.

29. Kleefstra SM, Zandbelt LC, de Haes HJ, et al. Trends in patient satisfaction in dutch university medical centers: room for improvement for all. BMC Health Serv Res 2015;15:112.

30. Chang CS, Chen SY, Lan YT. Service quality, trust, and patient satisfaction in interpersonal-based medical service encounters. BMC Health Serv Res 2013;13:22

31. Jean-Pierre P, Winters PC, Clark JA, et al. Do better-rated navigators improve patient satisfaction with cancer-related care? J Cancer Educ 2013;28:527-34.

32. Post DM, McAlearney AS, Young GS, et al. Effects of patient navigation on patient satisfaction outcomes. J Cancer Educ 2015;30:728-35.

33. Farley $\mathrm{H}$, Enguidanos ER, Coletti CM, et al. Patient satisfaction surveys and quality of care: an information paper. Ann Emerg Med 2014:64:351-7.

34. Anhang Price R, Elliott MN, Zaslavsky AM, et al. Examining the role of patient experience surveys in measuring health care quality. Med Care Res Rev 2014;71:522-54.

35. Browne K, Roseman D, Shaller D, et al. Analysis and commentary. Measuring patient experience as a strategy for improving primary care. Health Aff 2010;29:921-5.

36. Berkowitz $B$. The patient experience and patient satisfaction: measurement of a complex dynamic. Online J Issues Nurs 2016;21:1.

37. Goldzweig CL, Orshansky G, Paige NM, et al. Electronic patient portals: evidence on health outcomes, satisfaction, efficiency, and attitudes: a systematic review. Ann Intern Med 2013;159:677-87.

38. Footman K, Roberts B, Mills A, et al. Public satisfaction as a measure of health system performance: a study of nine countries in the former Soviet Union. Health Policy 2013;112:62-9.

39. Salisbury C, Wallace M, Montgomery AA. Patients' experience and satisfaction in primary care: secondary analysis using multileve modelling. BMJ 2010;341:c5004.

40. Swankoski KE, Peikes DN, Dale SB, et al. Patient experience midway through a large primary care practice transformation initiative. Am J Manag Care 2017;23:178-84.

41. Tung EL, Gao Y, Peek ME, et al. Patient experience of chronic illness care and medical home transformation in safety net clinics. Health Serv Res 2018;53:469-88.

42. Lehto U-S, Tenhola H, Taari K, et al. Patients' perceptions of the negative effects following different prostate cancer treatments and the impact on psychological well-being: a nationwide survey. $\mathrm{Br} J$ Cancer 2017;116:864-73. 